\title{
Upper urinary tract urothelial carcinoma with loco-regional nodal metastases: insights from the Upper Tract Urothelial Carcinoma Collaboration
}

\author{
Ramy F. Youssef, Shahrokh F. Shariat ${ }^{1}$, Yair Lotan, Christopher G. Wood ${ }^{2}$, \\ Arthur I. Sagalowsky, Richard Zigeuner ${ }^{3}$, Eiji Kikuchi ${ }^{4}$, Alon Weizer ${ }^{5}$, \\ Jay D. Raman ${ }^{1}$, Mesut Remzi ${ }^{6}$, Wareef Kabbani, Cord Langner ${ }^{3}$, \\ Charles C. Guo ${ }^{2}$, Marco Roscigno ${ }^{7}$, Francesco Montorsi ${ }^{7}$, Christian Bolenz ${ }^{8}$, \\ Wassim Kassouf ${ }^{9}$ and Vitaly Margulis \\ UT Southwestern Medical Center, Dallas, TX, ${ }^{1}$ Cornell University, New York, NY, ${ }^{2}$ UT M.D. Anderson Cancer Center, \\ Houston, TX, USA, ${ }^{3}$ Medical University of Graz, Graz, Austria, ${ }^{4}$ Keio University School of Medicine, Tokyo, Japan, \\ ${ }^{5}$ University of Michigan, Ann Arbor, MI, USA, ${ }^{6}$ Medical University of Vienna, Austria, ${ }^{7}$ Vita-Salute University, Milan, \\ Italy, ${ }^{8}$ Universitätsklinikum Mannheim, Mannheim, Germany, and ${ }^{9}$ McGill University, Montreal, Quebec, Canada \\ Accepted for publication 14 September 2010
}

Study Type - Therapy (multiinsititutional cohort) Level of Evidence $2 b$

\section{OBJECTIVE}

- To describe a multicentre experience with preoperative platinum-based chemotherapy before radical nephroureterectomy (RNU) in patients with upper tract urothelial carcinoma (UTUC) with loco-regional nodal metastases.

\section{PATIENTS AND METHODS}

- We identified 313 patients from the UTUC Collaboration (over 1200 patients), who underwent RNU with concomitant retroperitoneal lymph node dissection between 1990 and 2007 and met the inclusion criteria for one of three groups.

- Group 1 comprised patients who received chemotherapy before RNU because of biopsy-proven loco-regional nodal metastases.

- Group 2 consisted of patients who underwent primary RNU and were found to have metastatic nodal disease on final pathological review (node-positive).

What's known on the subject? and What does the study add?

Neoadjuvant chemotherapy offers survival benefits for patients with urothelial carcinoma of the bladder. However, it is still underutilized in the 'biologically similar' upper tract urothelial carcinoma. Systemic chemotherapy in a neoadjuvant setting is a more attractive option, as loss of renal function after nephrectomy can complicate the administration of adjuvant chemotherapy. We found that preoperative systemic therapy followed by aggressive surgical debulking is a promising treatment strategy for upper tract urothelial carcinoma patients with known or at risk of loco-regional nodal metastasis.

- Group 3 comprised a comparative cohort of patients treated with primary RNU for invasive or locally advanced (pT2/pT4) nodenegative (NO) UTUC.

\section{RESULTS}

- Groups 1, 2 and 3 included 18, 120 and 175 patients, respectively. The 5 -year disease-free survival rates were $49 \%$, $30 \%$ and $64 \%$, whereas the 5-year cancer-specific survival rates were $44 \%$, $36 \%$ and $69 \%$ in groups 1, 2 and 3, respectively.

- In group 1, on final pathological evaluation, nine patients were $\mathrm{pNO}$, six patients were pTO and five patients had pTONO disease. Kaplan-Meier survival analyses showed similar recurrence and survival rates in group 1 compared with group 3 ( $P=0.14$ and $P=0.06$, respectively).
- Meanwhile, group 2 had significantly lower disease-free and cancer-specific survival rates compared with group 3 $(P<0.001$ and $P<0.001$, respectively) and compared with group $1(P=0.04$ and $P=0.06$, respectively).

\section{CONCLUSIONS}

- Preoperative chemotherapy followed by aggressive surgical consolidation may yield favourable oncological outcomes in patients with UTUC with loco-regional nodal metastases.

- These data support further evaluation of neoadjuvant systemic therapy in patients at risk for locally advanced UTUC.

\section{KEYWORDS}

urothelial carcinoma, chemotherapy, lymph node metastases 


\section{INTRODUCTION}

Upper urinary tract urothelial carcinoma (UTUC) is relatively rare, accounting for approximately $5 \%$ of genitourinary malignancies, although recent data suggest that the incidence is increasing $[1,2]$. Radical nephroureterectomy (RNU) with excision of an ipsilateral bladder cuff via a laparoscopic or open approach remains the standard treatment in patients with invasive UTUC and a functional contralateral kidney. Although surgical techniques have improved, locally advanced UTUC still has a poor prognosis and systemic recurrences are common [2-4].

Unfortunately, with the rarity of the disease and historical difficulties in gathering patients with urothelial carcinoma for clinical trials, there are very few direct data providing clinical guidance on the optimal management of patients diagnosed with locally advanced UTUC. Small series show measurable response rates, specifically in patients treated with chemotherapy for metastatic or unresectable UTUC [5]. The loss of renal function that occurs with nephrectomy can complicate the administration of appropriate systemic chemotherapy in an adjuvant setting. Uncertainties regarding the survival benefit gained from chemotherapy combined with inadequacy of clinical staging are additional contributing factors that have led to the under-use of neoadjuvant chemotherapy for UTUC. However, analogous to the experience with urothelial carcinoma of the bladder (UCB), where administration of neoadjuvant cisplatin-based combination chemotherapy translated into a tangible survival advantage [6], it is argued that preoperative systemic chemotherapy should be used in patients with locally advanced UTUC $[6,7]$.

Patients with UTUC and loco-regional nodal metastatic disease represent a challenging cohort of patients, with no randomized trials to guide their optimal management. Using a multi-institutional database of patients treated for UTUC, we evaluated the oncological outcomes in patients with UTUC and loco-regional nodal metastases treated with preoperative systemic therapy followed by aggressive surgical consolidation with RNU. Oncological outcomes were compared with node-positive patients treated with immediate RNU and with node negative (NO) patients with locally advanced UTUC managed with immediate surgery.

\section{PATIENTS AND METHODS}

We used a database comprising patients from the UTUC Collaboration, this patient cohort has been described in detail elsewhere [3]. After combining the data sets from the enrolled centres, a database containing more than 1200 patients who underwent RNU with ipsilateral bladder cuff resection between 1987 and 2007 was generated. We identified 313 patients who met the criteria for one of three groups. Group 1 comprised patients who had clinical evidence of positive locoregional nodal metastases, confirmed by biopsy. These patients received preoperative chemotherapy followed by RNU. Group 2 consisted of patients who underwent primary RNU and were found to have metastatic nodal disease on final pathological review. Group 3 comprised a comparative cohort of patients treated with primary RNU for (pT2-T4NO) UTUC. Selection criteria required adequate pathological staging with standard, rather than limited, lymph node dissection during RNU. Adequate pathological nodal staging was defined as removal of the para-aortic, paracaval, or interaortocaval nodes from the renal hilum to the inferior mesenteric artery for renal pelvis and proximal ureteral tumours. For mid- and lower ureteral tumours, lymph node dissection was performed from the renal hilum to the bifurcation of the common iliac artery and ipsilateral pelvic nodes, respectively. The standardization of lymph node dissection was impossible because of the multicentre and retrospective study design. As a result of inadequate regional lymphadenectomy (as reported by individual contributing centres), 223 patients were excluded from further analysis.

All surgical specimens were processed according to standard pathological procedures, and all slides were re-reviewed by genitourinary pathologists according to prospectively defined uniform criteria. All pathologists were blinded to clinical outcomes. Tumours were staged according to the American Joint Committee on Cancer Union International Contre le Cancer (AJCCUICC) Tumor-Node-Metastasis (TNM) classification 2002 [8]. Tumour grading was assessed according to the $1998 \mathrm{WHO}$ International Society of Urologic Pathology (ISUP) consensus classification [9]. In addition, all specimens were evaluated for tumour location, pattern of tumour growth (papillary vs sessile), presence of lymphovascular invasion, tumour necrosis, and concomitant carcinoma in situ.

Follow up was performed according to institutional protocols. Patients were generally followed every 3 months for the first year after RNU and every 6 months from the second year. Follow up consisted of a history, physical examination, routine blood work and serum chemistry studies, urinary cytology, chest radiography, cystoscopic evaluation of the urinary bladder, and radiographic evaluation of the contralateral upper urinary tract. Elective bone scans, chest CT or MRI were taken when clinically indicated.

Statistical analysis was performed with SPSS version 17 (SPSS, Chicago, IL, USA). Bladder recurrences were not considered in the analysis of disease-free survival rate. Diseasefree survival and cancer-specific survival were estimated using the Kaplan-Meier survival analysis. Disease recurrence was defined as local failure in the nephroureterectomy bed, regional lymph nodes, or distant metastasis after RNU for UTUC. The period of diseasefree survival was defined as the time between the date of RNU and the development of local recurrence or distant metastasis. Censored survival values represent patients who were alive without clinical evidence of disease at the last follow-up. Cause of death was determined by the treating physicians, by chart review corroborated by death certificates, or by death certificates alone. The period of cancer-specific survival was defined as the time between the date of RNU and death due to cancer. Statistical differences were determined by the log-rank test. All reported $P$ values are two-sided and significance was set at $<0.05$.

\section{RESULTS}

Table 1 describes clinical characteristics of the patients according to the study grouping. Preoperative systemic chemotherapy regimens consisted of gemcitabine/ cisplatinum in 14 patients, methotrexate, vinblastine, doxorubicin and cisplatin (MVAC) in four patients. The patients in group 1 received two to nine preoperative chemotherapy cycles (median six cycles). Three of the 18 patients in this group, received additional adjuvant chemotherapy. Patients in groups 2 and 3 were managed with initial RNU (with open technique in 87\% 


\begin{tabular}{|c|c|c|c|c|}
\hline & $\begin{array}{l}\text { Group } 1 \\
\text { Preoperative } \\
\text { chemotherapy } \\
\text { before RNU }(\mathrm{N}+)\end{array}$ & $\begin{array}{l}\text { Group } 2 \\
\text { RNU }(\mathrm{N}+)\end{array}$ & $\begin{array}{l}\text { Group } 3 \\
\text { RNU (NO) }\end{array}$ & $\begin{array}{l}\text { Total } \\
\text { number of } \\
\text { patients }\end{array}$ \\
\hline Total, $n(\%)$ & $18(5.8)$ & $120(38.3)$ & $175(55.9)$ & $313(100)$ \\
\hline \multicolumn{5}{|l|}{ Gender, $n(\%)$} \\
\hline Female & $9(50)$ & $78(65)$ & $117(67)$ & $204(65)$ \\
\hline Male & $9(50)$ & $42(35)$ & $58(33)$ & 109 (35) \\
\hline \multicolumn{5}{|l|}{ Age, years } \\
\hline Mean + SD & $68 \pm 8$ & $68 \pm 12$ & $67 \pm 11$ & $68 \pm 11$ \\
\hline Range & $52-80$ & $27-90$ & $31-97$ & $27-97$ \\
\hline \multicolumn{5}{|l|}{ ECOG, $n(\%)$} \\
\hline 0 & $10(55.5)$ & $79(66)$ & $111(63)$ & $200(64)$ \\
\hline 1 & 7 (39) & $35(29)$ & $61(35)$ & $103(33)$ \\
\hline 2 & $1(5.5)$ & $6(5)$ & $3(2)$ & $10(3)$ \\
\hline \multicolumn{5}{|c|}{ Previous bladder UC diagnosis, $n(\%)$} \\
\hline Absent & $10(55.5)$ & $94(78)$ & $140(80)$ & $244(78)$ \\
\hline Present & $8(44.5)$ & $26(22)$ & $35(20)$ & $69(22)$ \\
\hline \multicolumn{5}{|l|}{ Surgical technique, $n(\%)$} \\
\hline Open & $17(94.5)$ & $104(87)$ & $150(86)$ & $271(86.5)$ \\
\hline Laparoscopic & $1(5.5)$ & $16(13)$ & $25(14)$ & $42(13.5)$ \\
\hline \multicolumn{5}{|l|}{ Chemotherapy, $n(\%)$} \\
\hline Neoadjuvant & $18(100)$ & 0 & 0 & $18(6)$ \\
\hline Adjuvant & $3(17)$ & $52(43)$ & $33(19)$ & $88(24)$ \\
\hline \multicolumn{5}{|l|}{ Outcome, $n(\%)$} \\
\hline Recurrence & $8(44)$ & $78(65)$ & $59(34)$ & $145(46)$ \\
\hline Death due to cancer & 7 (39) & $66(55)$ & $48(27)$ & $121(39)$ \\
\hline
\end{tabular}

ECOG, Eastern Cooperative Oncology Group; $N+$, node-positive; NO, node-negative; RNU, radical nephroureterectomy; UC, urothelial carcinoma.

FIG. 1. Disease-free survival after radical nephroureterectomy in 313 patients managed for advanced upper urinary tract urothelial carcinoma, according to treatment group.

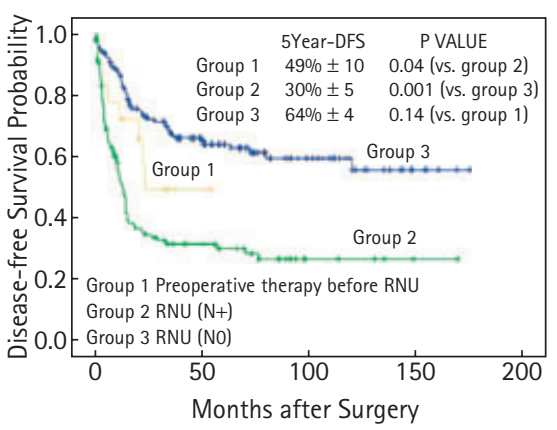

FIG. 2. Cancer-specific survival after radical nephroureterectomy in 313 patients managed for advanced upper urinary tract urothelial carcinoma, according to treatment group.

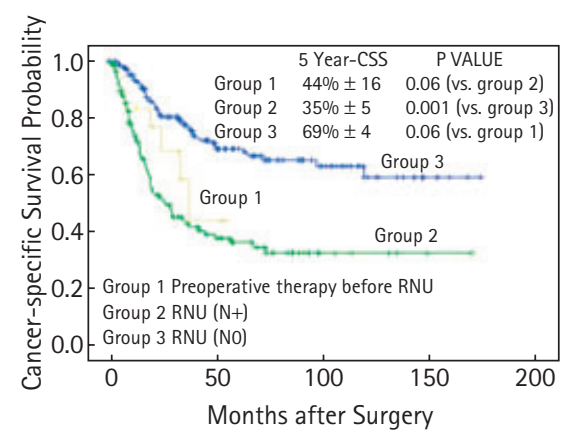

\section{DISCUSSION}

and $86 \%$ of cases, respectively) followed by adjuvant chemotherapy in 43\% and 19\% of patients, respectively. Adjuvant chemotherapy regimens consisted of MVAC in 60\%, gemcitabine/cisplatinum in 20\% and other platinum-based regimens in the remaining $20 \%$ of patients. On average, three cycles of adjuvant chemotherapy were administered (range one to six). Adjuvant systemic therapies were more likely to be used in younger patients as well as in patients with pathological features of advanced disease (pN1+, pT4Nany, pT3Nany). Table 2 describes the pathological characteristics in each group. In group 1, nine patients were pNO, six patients were pTO and five patients had pTONO disease on final pathological evaluation.

Figures 1 and 2 compare the probability of disease-free and cancer-specific survival among the study groups. The 5-year disease- free survival rates were $49 \%, 30 \%$ and $64 \%$, and the 5-year cancer-specific survival rates were 44\%, 36\% and 69\% in groups 1, 2 and 3, respectively. Kaplan-Meier survival analyses showed similar recurrence and survival rates in group 1 compared with group 3 ( $P=0.14$ and $P=0.06$, respectively). Meanwhile, group 2 had significantly lower disease-free survival and cancer-specific survival rates compared with group 3 $(P<0.001$ and $P<0.001$, respectively) and compared with group $1(P=0.04$ and $P=0.06$, respectively). Patients with no residual disease at RNU (pTOpNo) showed 83\% 5-year cancerspecific survival, compared with 31\% for patients with residual disease (pTany/pNany) $(P=0.092)$.

On multivariable Cox proportional hazards regression analysis, only pT stage $(P<0.001)$ and lymphovascular invasion $(P=0.003)$ were independent predictors of CSS.
UTUC and UCB share similar histological, biological, as well as molecular oncogenic characteristics and show comparable oncological outcomes when adjusted for tumour stage and grade $[6,7]$. Previous studies have shown a survival advantage in patients with UCB managed with neoadjuvant cisplatin-based combination chemotherapy before cystectomy $[6,10]$. Can this survival advantage be extrapolated to appropriately selected patients with UTUC? Unfortunately, conclusive data to support the use of neoadjuvant chemotherapy before RNU for UTUC are lacking. Moreover, unlike in UCB, clinical staging of patients with UTUC is of limited use, presenting additional difficulties in accurate selection of patients for neoadjuvant systemic therapy before RNU. Specifically, unlike in UCB, the depth of tumour invasion and primary tumour stage 
TABLE 2 Pathological characteristics of 313 patients managed for advanced upper urinary tract urothelial carcinoma, according to treatment group

\begin{tabular}{|c|c|c|c|c|}
\hline & $\begin{array}{l}\text { Group } 1 \\
\text { Preoperative } \\
\text { chemotherapy } \\
\text { before RNU (N+) }\end{array}$ & $\begin{array}{l}\text { Group } 2 \\
\text { RNU }(\mathrm{N}+)\end{array}$ & $\begin{array}{l}\text { Group } 3 \\
\text { RNU (NO) }\end{array}$ & $\begin{array}{l}\text { Total } \\
\text { number of } \\
\text { patients }\end{array}$ \\
\hline Total, $n(\%)$ & $18(5.8)$ & $120(38.3)$ & $175(55.9)$ & $313(100)$ \\
\hline \multicolumn{5}{|l|}{ Side, $n(\%)$} \\
\hline Right & 7 (39) & 47 (39) & $82(47)$ & $136(43.5)$ \\
\hline Left & $11(61)$ & $73(61)$ & $93(53)$ & $177(56.5)$ \\
\hline \multicolumn{5}{|l|}{ Index tumour location, $n(\%)$} \\
\hline Renal pelvis & $15(83.5)$ & $87(72.5)$ & $108(62)$ & $210(67)$ \\
\hline Ureter & $1(5.5)$ & $31(26)$ & $63(36)$ & $95(30)$ \\
\hline Uretero-enteric anastomosis & $2(11)$ & $2(1.5)$ & $4(2)$ & $8(3)$ \\
\hline \multicolumn{5}{|l|}{ Tumour architecture, $n(\%)$} \\
\hline Papillary & $3(17)$ & $53(44)$ & $115(66)$ & $171(55)$ \\
\hline Sessile & $15(83)$ & $67(56)$ & $60(34)$ & $142(45)$ \\
\hline \multicolumn{5}{|l|}{ Tumour grade, $n(\%)$} \\
\hline Low & $2(11)$ & $3(2.5)$ & $40(23)$ & 45 (14.5) \\
\hline High & $16(89)$ & $117(97.5)$ & $135(77)$ & $268(85.5)$ \\
\hline \multicolumn{5}{|l|}{ Pathological T stage, $n(\%)$} \\
\hline T0 & $6(33)$ & $0(0)$ & $0(0)$ & $6(2)$ \\
\hline $\mathrm{T} 1$ & $0(0)$ & $6(5)$ & $0(0)$ & $6(2)$ \\
\hline T2 & $3(17)$ & $19(16)$ & $61(35)$ & $83(26)$ \\
\hline T3 & 7 (39) & $66(55)$ & $108(62)$ & $181(58)$ \\
\hline $\mathrm{T} 4$ & $2(11)$ & $29(24)$ & $6(8)$ & $37(12)$ \\
\hline \multicolumn{5}{|l|}{ Concomitant $\mathrm{CIS}, n(\%)$} \\
\hline Absent & $11(61)$ & $70(58)$ & $116(66)$ & $197(63)$ \\
\hline Present & 7 (39) & $50(42)$ & $59(34)$ & 37 (37) \\
\hline \multicolumn{5}{|l|}{ Necrosis, $n(\%)$} \\
\hline Absent & $10(55.5)$ & $53(44)$ & $115(66)$ & $178(57)$ \\
\hline Present & $8(44.5)$ & $67(56)$ & $60(34)$ & $135(43)$ \\
\hline \multicolumn{5}{|l|}{$N$ stage, $n(\%)$} \\
\hline 0 & $9(50)$ & $0(0)$ & $175(100)$ & $184(59)$ \\
\hline 1 & $4(22)$ & $94(78)$ & $0(0)$ & $98(31)$ \\
\hline 2 & $5(28)$ & $26(22)$ & $0(0)$ & $31(10)$ \\
\hline Mean no. of removed LNs (range) & $13(1-35)$ & $6(1-41)$ & $7(1-27)$ & $7(1-41)$ \\
\hline Mean no. of positive LNs (range) & $1(0-10)$ & $3(1-22)$ & $0(0)$ & $1(0-22)$ \\
\hline \multicolumn{5}{|l|}{$\mathrm{LVI}, n(\%)$} \\
\hline Absent & $11(61)$ & $35(29)$ & $106(61)$ & $152(48.5)$ \\
\hline Present & 7 (39) & $85(71)$ & 69 (39) & $161(51.5)$ \\
\hline
\end{tabular}

CIS, carcinoma in situ; LN, lymph nodes; LVI, lymphovascular invasion; N+, node-positive; NO, nodenegative; $R N U$, radical nephroureterectomy.

are difficult to ascertain with delicate endoscopic instruments designed for the upper urinary tract. In an ongoing Phase II clinical trial of neoadjuvant systemic therapy for UTUC, all patients with high-grade UTUC are offered systemic chemotherapy before surgery, because of the high correlation between high tumour grade and advanced pathological stage [11]. Alternatively, we have recently developed a preoperative nomogram, using grade, architecture and location of the tumour to predict the probability of nonorgan-confined UTUC [12]. If validated prospectively, this tool can be used for selection of patients to receive systemic therapy before RNU.

The results of this study suggest that favourable oncological outcomes can be achieved in patients with UTUC and locoregional nodal metastases treated with preoperative systemic therapy followed by aggressive surgical consolidation with RNU. This is shown by a significant rate of complete pathological responses achieved in both primary tumours and lymph nodes, as well as by the favourable survival observed in nodepositive patients treated with preoperative chemotherapy before consolidative RNU, compared with node-positive patients treated with initial surgery. Ultimately, the safety and efficacy of such an approach should be evaluated in the context of a prospective clinical trial [11].

In UCB, the advantages of neoadjuvant chemotherapy include timely treatment of systemic micrometastatic disease; significant rates of complete pathological responses in the bladder and decrease in positive surgical margins [1]. In UTUC, several unique disease features lend additional rationale for the use of neoadjuvant, rather than the adjuvant, chemotherapy in these patients. The loss of renal function that occurs with RNU can significantly complicate administration of appropriate adjuvant chemotherapy after surgery, especially in the elderly patient population, who are likely to have a multitude of associated comorbidities. Hence, the additional advantages of administering chemotherapy before RNU include better treatment tolerance and the ability to deliver higher treatment doses than in the adjuvant setting [7]. Despite concerns about increased surgical morbidity after preoperative chemotherapy, the data in patients with UCB have not supported this idea $[13,14]$. Proposed benefits of preoperative chemotherapy have to be carefully balanced against the risks of overtreatment and delay of potentially curative surgical intervention. Isolated clinical pathological parameters, such as tumour stage and grade, are not adequate for accurate assessment of the true disease burden so integrative staging tools, similar to a recently developed preoperative nomogram for prediction of non-organ-confined UTUC can guide patient selection for neoadjuvant chemotherapy [12].

Unfortunately, our data suggest that systemic chemotherapy continues to be underutilized in patients with UTUC, with $<50 \%$ of node-positive patients receiving chemotherapy before or after RNU. The reasons for this underutilization remain unclear, but probably include physician practice patterns, unwillingness by patients to undergo chemotherapy, and significant deterioration of renal function after RNU. 
Based on our findings, as well as on experience gained from the management of patients with advanced UCB, preoperative platinum-based chemotherapy should be prospectively explored and incorporated into treatment algorithms of patients with advanced UTUC. We believe that an aggressive strategy with preoperative chemotherapy followed by RNU including meticulous lymph node dissection [15-17] may improve the outcome of these patients.

The data presented here are descriptive and exploratory, and several limitations of this study merit further discussion. First, are the limitations inherent to the retrospective study design, which undoubtedly translate into a significant selection bias and a disproportionally small number of patients in the preoperative chemotherapy group. Moreover, the number of patients treated with preoperative chemotherapy that could undergo subsequent RNU because of disease progression or declining performance status is not known. In addition, the true number of patients with clinically positive regional lymphadenopathy who did not undergo diagnostic biopsy and were managed with chemotherapy and surgery is not known. Finally, the patients with UTUC who were included in this study were managed by multiple surgeons who used different surgical techniques and templates of lymph node dissection. However, there was no established standard of care with regard to the performance and the extent of lymph node dissection during RNU at the time of study [18]. We believe that, with the rarity of UTUC, a retrospective study design, in which rigorous clinical and pathological review of patient data from multiple high-volume cancer centres is implemented, provides valuable clinical information, and serves as an important link into thoughtful prospective clinical trial design.

Preoperative systemic therapy followed by aggressive surgical consolidation may afford favourable oncological outcomes in patients with UTUC with loco-regional nodal metastases. This approach represents a promising treatment strategy for patients with UTUC with known loco-regional nodal metastasis or for those at risk of such metastases and should be explored in prospective protocols.

\section{ACKNOWLEDGEMENTS}

We want to thank members from the Upper Tract Urothelial Carcinoma Collaborative Group who participated in this study: Shuji Mikami, Takeo Kosaka, Masaru Isida, Keio University School of Medicine, Japan; Andrea Haitel, University of Vienna, Vienna, Austria; Roberto Bertini, Vita-Salute University, Milan, Italy; Philipp Stroebel, Maurice Stephan Michel, Universitätsklinikum Mannheim, Mannheim, Germany; Casey K. Ng, Douglas S. Scherr, Yingbei Chen, Cornell University, NY, USA; Mario A. Fernández, Clínica Alemana de Santiago, Chile; and Jeffrey Wheat, J. Stuart Wolf, Jr., University of Michigan, MI, USA

\section{CONFLICT OF INTEREST}

None declared.

\section{REFERENCES}

1 Raman JD, Scherr DS. Management of patients with upper urinary tract transitional cell carcinoma. Nat Clin Pract Urol 2007; 4: 432-43

2 Zigeuner R, Pummer K. Urothelial carcinoma of the upper urinary tract: surgical approach and prognostic factors. Eur Urol 2008; 53: 720-31

3 Margulis V, Shariat SF, Matin SF et al. Outcomes of radical nephroureterectomy: a series from the Upper Tract Urothelial Carcinoma Collaboration. Cancer 2009; 115: 1224-33

4 Koukourakis G, Zacharias G, Koukourakis $\mathrm{M}$ et al. Comprehensive management of upper tract urothelial carcinoma. Adv Urol 2009; doi:10.1155/ 2009/656521

5 Tannock I, Gospodarowicz M, Connolly J et al. M-VAC (methotrexate, vinblastine, doxorubicin and cisplatin) chemotherapy for transitional cell carcinoma: the Princess Margaret Hospital experience. J Urol 1989; 142: 289-92

6 Grossman HB, Natale RB, Tangen CM et al. Neoadjuvant chemotherapy plus cystectomy compared with cystectomy alone for locally advanced bladder cancer. N Engl J Med 2003; 349: 859-66

7 O'Donnell PH, Stadler WM. The role of chemotherapy in upper tract urothelial carcinoma. Adv Urol 2009; doi:10.1155/2009/419028

8 Greene FL, Page DL, Fleming ID et al. American Joint Committee on Cancer
(AJCC) Staging Manual, 6th edn Philadelphia: Springer, 2002

9 Epstein Jl, Amin MB, Reuter VR, Mostofi FK. The World Health Organization/International Society of Urological Pathology consensus classification of urothelial (transitional cell) neoplasms of the urinary bladder. Bladder Consensus Conference Committee. Am J Surg Pathol 1998; 22: 1435-48

10 Winquist E, Kirchner TS, Segal R, Chin J, Lukka H. Neoadjuvant chemotherapy for transitional cell carcinoma of the bladder: a systematic review and meta-analysis. J Uro/ 2004; 171: 561-9

11 Matin SF, Margulis V, Kamat A et al. Incidence of downstaging and complete remission after neoadjuvant chemotherapy for high-risk upper tract transitional cell carcinoma. Cancer 2010; 116: 3127-34

12 Margulis V, Youssef RF, Karakiewicz PI et al. Preoperative multivariable prognostic model for prediction of nonorgan confined urothelial carcinoma of the upper urinary tract. J Urol 2010; 184: 453-8

13 Millikan R, Dinney C, Swanson D et al. Integrated therapy for locally advanced bladder cancer: final report of a randomized trial of cystectomy plus adjuvant M-VAC versus cystectomy with both preoperative and postoperative M-VAC. J Clin Oncol 2001; 19: 4005-13

14 Hall MC, Swanson DA, Dinney CP. Complications of radical cystectomy: impact of the timing of perioperative chemotherapy. Urology 1996; 47: 826-30

15 Brausi MA, Gavioli M, De Luca G et al. Retroperitoneal lymph node dissection (RPLD) in conjunction with nephroureterectomy in the treatment of infiltrative transitional cell carcinoma (TCC) of the upper urinary tract: impact on survival. Eur Urol 2007; 52: 1414-8

16 Roscigno M, Cozzarini C, Bertini R et al. Prognostic value of lymph node dissection in patients with muscleinvasive transitional cell carcinoma of the upper urinary tract. Eur Urol 2008; 53 : 794-802

17 Roscigno M, Shariat SF, Margulis V et al. Impact of lymph node dissection on cancer specific survival in patients with upper tract urothelial carcinoma treated with radical nephroureterectomy. J Urol 2009; 181: 2482-9 
METASTATIC UPPER URINARY TRACT UROTHELIAL CARCINOMA

18 Capitanio U, Shariat SF, Isbarn H et al. Comparison of oncologic outcomes for open and laparoscopic nephroureterectomy: a multiinstitutional analysis of 1249 cases. Eur Urol 2009; 56: 1-9
Correspondence: Vitaly Margulis, Department of Urology, The University of Texas

Southwestern Medical Center, 5323 Harry

Hines Boulevard, Dallas, TX 75390-9110,

USA.

e-mail: Vitaly.Margulis@utsouthwestern.edu
Abbreviations: UTUC, Upper urinary tract urothelial carcinoma; $\mathrm{RNU}$, radical nephroureterectomy; UCB, urothelial carcinoma of the bladder; MVAC, methotrexate, vinblastine, doxorubicin and cisplatin. 